Canad. Math. Bull. Vol. 22 (2), 1979

\title{
ON THE POSITIVE DEFINITENESS OF A FUNCTIONAL
}

\author{
J. N. PANDEY
}

\begin{abstract}
In this note, we have shown that the set of conditions given by Gel'fand and Vilenkin for the positive definiteness of a functional $L(\phi)$ [1, Theorem 7, p. 285] though sufficient is not necessary, thereby providing an answer to an open problem posed by them [1, Theorem 7 , p. 285].
\end{abstract}

The notation and terminology of this work will follow those of [1]. The set of infinitely differentiable complex-valued functions defined over real line with compact support will be denoted by $K$. A functional $L(\phi)$ defined for all $\phi_{p} \in K$ will be said to be positive definite if

$$
\sum_{l, m=1}^{p} L\left(\phi_{l}-\phi_{m}\right) \xi_{l} \bar{\xi}_{m} \geq 0
$$

for all $\phi_{1}, \phi_{2}, \ldots \phi_{p}$ belonging to $K$ and complex numbers $\xi_{1}, \xi_{2}, \ldots, \xi_{p}$. Likewise, a function $f(x)$ defined over the real line will be said to be positive definite if

$$
\sum_{l, m=1}^{p} f\left(x_{l}-x_{m}\right) \xi_{l} \bar{\xi}_{m} \geq 0
$$

for all real $x_{1}, x_{2}, \ldots, x_{p}$ and complex $\xi_{1}, \xi_{2}, \ldots, \xi_{p}$. Similarly, we can extend the notion of positive definiteness to a function of $p$ real variables.

Description of the problem. Gel'fand and Vilenkin [1, Theorem 2, p. 275] have shown that in order that the functional $L(\phi)$ defined by

$$
L(\phi)=\exp \left(\int f[\phi(t)] d t\right) \quad \forall \phi \in K
$$

where $f(x)$ is a continuous function satisfying $f(0)=0$ be positive definite it is necessary and sufficient that the function $e^{s f(x)}$ be positive definite for all

Received by the editors March 1st, 1978.

AMS Subject classification

Primary: 46F10, 46E40

Secondary: $60 \mathrm{~J} 30$

Key words and phrases: Positive definite functional, positive definite function. Generalized Stochastic Process, Stochastic processes with independent values at every point. 
positive values of the parameter $s$. The importance of this theorem lies in characterization of generalized Stochastic processes with independent values at every point. They, however, attempted to prove similar theorem for a functional

$$
L(\phi)=\exp \left(\int_{-\infty}^{\infty} f\left(\phi, \phi^{\prime}, \phi^{\prime \prime}, \ldots, \phi^{(n)}\right) d t\right) \quad \forall \phi \in K
$$

where $f\left(x_{0}, x_{1}, x_{2}, \ldots, x_{n}\right)$ is a continuous function of $(n+1)$ variables with $f(0,0, \ldots, 0)=0$. They affirm $[1$, Theorem 7, p. 285] that in order that the functional

$$
L(\phi)=e^{M(\phi)} \quad \forall \phi \in K
$$

where

$$
M(\phi)=\int_{-\infty}^{\infty} f\left[\phi, \phi^{\prime}, \phi^{\prime \prime}, \ldots \phi^{(n)}\right] d t
$$

be positive-definite, it is sufficient that for any $s>0$, the function $\exp \left[s f\left(x_{0}, x_{1}, x_{2}, \ldots x_{n}\right)\right]$ be positive definite function of the variables $x_{0}, x_{1}, x_{2}, \ldots x_{n}$. They also stated that it is not known if the above condition is also necessary for the positive definiteness of $L(\phi)$ defined in Eqn. (3). In this note, we have proved by giving a counter example that the above-mentioned condition is not at all necessary for the positive definiteness of the functional $L(\phi)$ defined by (3). We have also given a neecessary condition for the positive definiteness of the functional $L(\phi)$ defined by (3).

Counter example. With $n=1$, let us define

$$
f\left(x, x_{0}\right)=i x_{0}+x_{0} x_{1} \text { where } i=\sqrt{ }-1 .
$$

Define

$$
\begin{aligned}
L(\phi) & =\exp \left[\int_{-\infty}^{\infty} f\left(\phi, \phi^{\prime}\right) d t\right] \quad \forall \phi \in K \\
& =\exp \left[i \int_{-\infty}^{\infty} \phi(t) d t\right]
\end{aligned}
$$

as

$$
\int_{-\infty}^{\infty} \phi(t) \phi^{\prime}(t) d t=0
$$

The functional $L$ defined over $K$ by the relation

$$
L(\phi)=\exp \left[i \int_{-\infty}^{\infty} \phi(t) d t\right]
$$


is positive definite as the function $e^{i s x}$ is positive definite for each $s>0$. (See [1, Theorem 2, p. 275]). In fact, for real $x_{1}, x_{2}, \ldots, x_{p}$, the determinant of the matrix $\left[a_{l, m}\right]_{p}$ with $a_{l, m}=e^{i s\left(x_{1}-x_{m}\right)}$ is zero. Since the matrix $\left[a_{l, m}\right]_{p}$ is Hermitian it follows that the function $e^{i s x}$ is positive definite. Therefore, the functional $L(\phi)$ defined by (4) is also positive definite. But the function $\exp \left[s\left(i x_{0}+x_{0} x_{1}\right)\right]$ for each $s>0$ is not positive definite as the following manipulation shows for $p=2$.

Let us take $\left.a_{l, m}=\exp \left(s\left[x_{1}-x_{m}\right) i+\left(x_{l}-x_{m}\right)\left(y_{l}-y_{m}\right)\right]\right)$ where $l, m=1,2$ the value of the determinant of the matrix $\left[a_{l, m}\right]$ is $=1-e^{2 s\left(x_{1}-x_{2}\right)\left(y_{1}-y_{2}\right)}$ which can assume negative values for $x_{1}>x_{2}$ and $y_{1}>y_{2}$.

Therefore, the conditions stated by Gel'fand and Vilenkin in [1, Theorem 7, p. 285] for the positive definiteness of the functional $L$ given by (2) is only sufficient and is not at all necessary. We are, however, stating below a condition which is necessary for the positive definiteness of the functional $L$ as defined by (2) but is not sufficient either.

TheOREM. Let $\phi(t) \in K$. Assume that $f\left(x_{0}, x_{1}, x_{2}, \ldots, x_{n}\right)$ is a continuous function of $(n+1)$ variables $x_{0}, x_{1}, \ldots, x_{m}$ such that $f(0,0,0 \cdots 0)=0$. Define

$$
L(\phi)=e^{M(\phi)},
$$

where

$$
M(\phi)=\int f\left(\phi, \phi^{\prime}, \phi^{\prime \prime}, \ldots \phi^{(n)}\right) d t
$$

Then a necessary condition for the functional $L(\phi)$ to be positive definite is that the function $e^{s(f(x, 00, \ldots 0))}$ be a positive definite function for each $s>0$.

Proof. Define a function $g_{i}(x)$ for each $i=1,2, \ldots p$ such that

$$
g_{i}(t)= \begin{cases}x_{i} & \text { when } 0<t<s \\ 0 & \text { elsewhere }\end{cases}
$$

Let $\left\{\phi_{i, \nu}\right\}_{\nu=1}^{\infty}$ be a sequence of functions in $K$ converging to the step function $g_{i}(t)$ uniformly a.e. for each fixed $i=1,2,3, \ldots p$. By assumption we have

$$
\sum_{i, j=1}^{p} L\left(\phi_{i, \nu}-\phi_{j, \nu}\right) \xi_{i} \bar{\xi}_{j} \geq 0
$$

Our result now follows by letting $\nu \rightarrow \infty$ in (6).

Incidentally, for examples of positive definite functions, one can refer to $[2$, p. 95] and [3, p. 357].

Acknowledgement. The author expresses his gratefulness to Professor P. R. Beesack of our department who was readily available for consulation.

The work of the author was supported by the National Research Council, Grant No. A5298. 


\section{REFERENCES}

1. Gel'fand I. M. and Vilenkin, N. Ya, Generalized functions, Volume 4, 1964 Academic Press.

2. Hirschman, I. I. and Widder, D. V., The convolution transform, 1955, Princeton University Press.

3. Karlin, Samuel, Total positivity, Volume I, 1968, Stanford University Press.

Centre de Recherches Mathématiques

UNIVERSITÉ DE MONTRÉAL

Montréal, QuÉ. H3C 3J7

\author{
DEPARTMENT OF MATHEMATICS \\ CARLETON UNIVERSITY \\ OTtaWA, ONT. K1S 5B6
}

